\title{
Antibodies, introns and biosynthetic versatility
}

\author{
from Miranda Robertson and Mike Hobart
}

Soon after the discovery that eukaryotic genes may be interrupted by non-coding DNA sequences, Gilbert ${ }^{1}$ speculated in Nature that this unexpected arrangement might make it possible to produce variants of a single protein by differential splicing of the interrupted RNA. Specifically, he suggested that such a mechanism might underlie the well defined sequential changes that take place in the immunoglobulin molecule in the course of B lymphocyte ontogeny. As a result of these changes, a single antigen-binding site is associated successively with different effector sites to produce first immunoglobulin $\mathrm{M}$, then immunoglobulin $\mathrm{D}$, and later immunoglobulin $\mathrm{G}$ or sometimes $\mathrm{A}$ or E. Each of the different classes of immunoglobulin is expressed first as a membranebound receptor molecule and later, on the terminal differentiation of the lymphocyte, as a secreted protein which is identical to the receptor in all but a few residues at its carboxy terminal.

Both the class of the immunoglobulin molecule and whether it is membranebound or secreted depend on the structure of the constant region of its heavy chains (see Fig. 1). Thus each heavy-chain variable region $\left(\mathrm{V}_{\mathrm{H}}\right.$ region) is associated in turn with a constant region $\left(\mathrm{C}_{\mathrm{H}}\right.$ region) coding for the $\mu, \delta$ and $\gamma, \alpha$ or $\varepsilon$ chains corresponding to IgM, IgD, IgG, IgA and IgE; and each of these chains may then undergo modification at its $3^{\prime}$ end. It is now known that the class switch from IgM to $\operatorname{IgG}, \operatorname{IgA}$ or $\operatorname{IgE}$ is mediated by translocation of the $\mathrm{V}_{\mathrm{H}}$ region DNA with deletion of the $C_{H}$ regions $5^{\prime}$ to the one that is expressed ${ }^{2}$. Deletion, however, cannot explain the switch from IgM to IgD, because some B cells, including a few tumour cell lines, synthesize $\operatorname{IgM}$ and $\operatorname{IgD}$ at the same time; nor is it an attractive explanation for the switch from membrane to secreted proteins,

Miranda Robertson is an associate editor of Nature and Mike Hobart is at the Medical Research Council Centre, Cambridge.

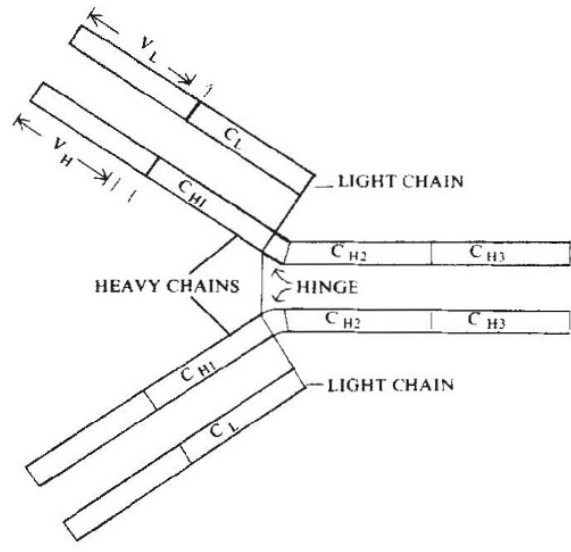

Fig.1 Diagram of an antibody molecule showing the division of the heavy chains into the variable regions that determine antigenbinding specificity and the constant region domains. Each of the four domains, $\mathrm{C}_{\mathrm{H} 1}$, $\mathrm{C}_{\mathrm{H} 2}, \mathrm{C}_{\mathrm{H} 3}$ and the hinge, is encoded in a separate intron on the DNA. The sequences of the $C_{H}$ introns are different for the different classes of immunoglobulin. The secreted form of each class $\left(C_{s}\right)$ differs from the membrane form $\left(C_{m}\right)$ in the last few carboxy terminal residues of $\mathrm{C}_{\mathrm{H} 3}$ : the membrane form has a longer and more hydrophobic carboxyterminal which anchors it in the membrane;

the diagram shows the secreted version.

which are also simultaneously expressed. Instead, as Gilbert surmised, these processes are controlled by processing of the RNA.

Immunoglobulin D biosynthesis has become accessible to investigation through the work of Liu and his colleagues ${ }^{3}$ who have located the gene coding for the corresponding $\delta$ constant region. The gene itself is very peculiar, having only two constant region domains (instead of the usual three) and an abnormal hinge region. Furthermore, it lies only about 2,000 base pairs from the $\mu$ gene corresponding to the IgM constant region; this is a much shorter distance than has so far been found to separate other constant region genes, and it means a single stable transcript could be made from the combined $\mu$ and $\delta$ coding regions. There is, indeed, now preliminary evidence for the existence of such a transcrip $t^{4}$. The switch from $\mu$ to $\delta$ could thus be mediated by the removal of the entire $\mu$ gene as an intron (see Fig.2). The simultaneous expression of $\mu$ would be due to simultaneous production of shorter transcripts, ending before the $\delta$ sequences, or to polyadenylation at a site between the $\mu$ and $\delta$ genes.

This scheme has more recently been strongly reinforced by Moore et al., who have confirmed the location of the $\delta$ gene in DNA both from mouse sperm and from a mouse hybridoma expressing membranebound forms of $\operatorname{IgM}$ and $\operatorname{IgD}$, and established that the $\mu$ gene is not rearranged in the hybridoma $\mathrm{DNA}^{5}$. At the same time, however, they have raised the possibility that plasma cells committed to the secretion of $\mathrm{IgD}$, unlike B cells transiently synthesizing the membrane form, may delete the $\mu$ sequences. So far, the evidence rests on a single rat plasmacytoma which secretes IgD and seems to have deleted the $\mu$ gene. Whether the deletion of $\mu$ is really a normal concomitant of the terminal differentiation of IgD-secreting lymphocytes, or whether it simply reflects the notorious instability of constant region genes in cultured cells, it is too early to say. It is not yet known, for example, whether the specialized switch sequences characteristic of the regions on the $5^{\prime}$ side of the $\mu$ and $\gamma$ genes $^{2}$ are to be found between the mouse $\mu$ and $\delta$ genes; but Rabbitts et al. ${ }^{6}$ have sequenced most of the region between the human $\mu$ and $\delta$ genes without discovering any.

The deletion or otherwise of the $\mu$ gene has, however, no direct bearing on the switch from membrane to secreted IgD, which is presumed to occur by the mechanism recently established for IgM. Independent observations by Alt et al. ${ }^{7}$ and Rogers et al. ${ }^{8}$ show that cells which are making both membrane and secreted IgM

Fig.2 Diagram of chromosomal DNA coding for IgM and $\operatorname{IgD}$ in a $\mathrm{B}$ cell. The open rectangles are exons and the intervening solid lines represent introns. Heavy vertical bars represent RNA splicing signals. In cells expressing both membrane IgM and membrane IgD, it is believed that the entire region is transcribed. Dotted line $a$ joins the introns that are spliced to produce membrane $\operatorname{IgD}$, with the elimination of all the $\mathrm{C} \mu$ introns. Dotted line $b$ joins the introns that are spliced to produce the membrane form of IgM. Note that if the switch from membrane to secreted IgM occurs through termination of transcription after the $\mu_{\mathrm{s}}$ segment, transcription of the $\delta$ gene will also be shut off. The switch from membrane to secreted $\delta$ chains is believed to occur as described for $\mu$.

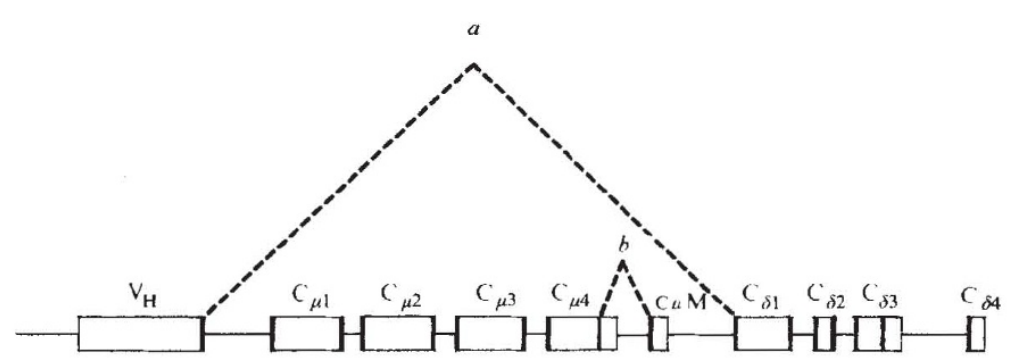


contain $\mu$ chain messenger RNAs of different lengths. By examining cells in which the two forms of $\mu$ chain are synthesized in different proportions, Alt et al. were able to deduce that the longer of the two messages codes for the membrane $\left(\mu_{m}\right)$ form. Rogers et al. hybridized the two messages with cloned genomic DNA containing the $\mu$ gene, and found that while both hybridize the coding sequence of the four major $\mu$ domains, the longer one also hybridizes with a short segment on the $3^{\prime}$ side. The same team went on to show ${ }^{9}$ that this short segment codes for a hydrophobic protein sequence whose characteristics are similar to those of known membranebound proteins.

Thus the secreted form of the protein $\left(\mu_{\mathrm{s}}\right)$ is encoded by the four main $\mu$ gene segments, while the expression of the membrane form entails the addition of a further segment at the $3^{\prime}$ side. This $3^{\prime} \mu_{\mathrm{m}}$ segment has a sequence characteristic of a splice site at the beginning of an exon at its $5^{\prime}$ end; but the matching splice sequence upstream occurs within the coding sequence of the $\mathrm{C} \mu 4$ domain, followed by the coding sequence for the last 19 amino acids of the $\mu_{\mathrm{s}}$ chain and a termination codon. Early et al. suggest on the basis of this organization that $\mu_{m}$, the first antibody to be synthesized by a B cell, is produced from a long transcript extending to a polyadenylation site beyond the $\mu_{\mathrm{m}}$ coding segment. In the course of the processing of the transcript to mRNA the $\mu_{\mathrm{s}}$ coding segment is eliminated as an intron, with the consequence that the termination codon is also removed.

When the $B$ cell matures into an antibody-secreting plasma cell, either transcription terminates upstream of the $\mu_{\mathrm{m}}$ segment or, for some reason, the $\mu_{\mathrm{m}}$ segment is cleaved from the transcript. In either case the $\mu_{s}$ sequence is retained in the mRNA and read until the termination codon.

Thus the class switch from $\operatorname{IgM}$ to $\operatorname{IgD}$ seems to work by a mechanism analogous to that which operates the switch from $\mu_{\mathrm{m}}$ to $\mu_{\mathrm{s}}$; and it has, incidentally, been pointed out that the $\mu_{m}-\mu_{s}$ switch may automatically eliminate the production of IgD by IgM-secreting cells if transcription ends at the $\mu_{\mathrm{s}}$ segment (see Fig.2).

The organization and regulation of the $\delta$ coding sequences open two further questions, the first specifically about the $\delta$ chain and the second generally about RNA splicing.

The first arises from the fact that the

1. Gilbert, W. Nature 271, 501 (1978)

2. see Molgaard, H. Nature 286, 657 (i980).

3. Lin, C.P., Tucker, P.W., Mushinski, F.J. \& Blattner, F. R. in, C.P., Tucker, P.W., M
Science 209, 1348 (1980).

Wall, R. et al. J. supramolec. Struct. \& cell. Biochem. Suppl. 5, abstract 5 (1981)

5. Moore, R.W. et al. Proc. natn. Acad. Sci. U.S.A. (in the press).

6. Rabbits, T.J. J. supramolec. Struct. \& cell. Biochem. Suppl. 5, abstract 30 (1981)

Ait, F.W. et al. Cell 20, $293(1980)$

All, F.W. et al. Cell 20، $293(1980)$

Rogers, J. et al. Cell 20, 303 (1980)

Corte, G. et al Scand. J. Immunol.

1. Maki, R. et al. Cell (in the press). coding sequences as described by Liu et al. can account for the shorter of the two known versions of the $\delta$ chain but not for the longer version ${ }^{10}$. However, further sequences apparently coding for the $\delta$ chain have more recently been detected (but not yet characterized) ${ }^{11}$ further downstream, lending weight to the proposal of Liu et al. that differential splicing may provide a family of different $\delta$ chains with different - and it must be confessed entirely unknown - functions.

The second question is that of the mechanism by which introns are eliminated from nuclear RNA. Until now, the simplest scheme - that the splice site at the end of each exon is paired with the matching sequence at the beginning of the next one downstream - has also been consistent with the known data. However, it plainly cannot account for the removal of the $\mu$ exons from the large $\mu-\delta$ transcript. There is still a formal possibility that such a combined transcript does not exist; but if it does, it will clearly represent a further strong incentive to molecular geneticists of immunoglobulin to devise a splicing sytem that will work in vitro.

\section{Anomalous sputtering at high energy}

\section{from P.K. Haff and L.E. Seiberling}

WHENEVER a sufficiently energetic stream of particles impinges on a target, there is some probability that target atoms and molecules will be knocked loose from the surface. This process of erosion on an atomic scale is called sputtering. For incident ions in the $\mathrm{keV}$ per AMU range, the average number of target particles ejected per ion is called the sputtering yield, $S$. Values of $S$ in the range 1 to 10 are common. Quantitative calculations of the sputtering yield in this regime are based on the idea of a collision cascade (a primary recoil target atom makes collisions which produce secondaries which in turn produce tertiaries, and so on), and predicted yields typically agree with experiment to within a factor of 2 or 3 .

At higher-incident ion energies, in the hundreds of $\mathrm{keV}$ per AMU to several $\mathrm{MeV}$ per AMU range, for example, the collision cascade predictions for the sputtering yield are small compared with unity. However, recent experiments using high-energy ion beams have shown that for some materials very large sputtering yields ( $\sim 4,000$ in some cases) exist when the collision cascade prediction is $S \ll 1$.

At Bell Laboratories, Walter Brown and his collaborators ${ }^{1,2}$ have shown that $\mathrm{H}$ and $\mathrm{He}$ in the $100 \mathrm{keV}$ per AMU range can sputter $\mathrm{H}_{2} \mathrm{O}$ ice at rates up to three orders of magnitude faster than those predicted according to the low-energy collision cascade calculations. Moreover, at temperatures below $-100 \mathrm{~K}$, the yield is independent of temperature, and appears to be proportional to the square of the electronic stopping power, $(\mathrm{d} E / \mathrm{d} x)_{e}^{2}$. This is to be contrasted with the energy dependence of the collision cascade, which is given by the nuclear stopping power $(\mathrm{d} E / \mathrm{d} x)_{n}$ describing the energy loss of the projectile to elastic atomic collisions. Evidently energy is transferred from the initial electronic excitation into kinetic energy of atoms, some of which escape as sputtered particles. Brown and his colleagues favour an explanation based on the ion explosion model. Neighbouring atoms, partially stripped of their electrons by the passage of the projectile, acquire kinetic energy through their mutual Coulomb repulsion. Such a picture leads essentially to a $(\mathrm{d} E / \mathrm{d} x)_{e}^{2}$ prediction for the energy dependence.

An early prediction ${ }^{3}$, that in certain dielectrics the ion explosion mechanism would lead to an anomalously high sputtering yield essentially proportional to $(\mathrm{d} E / \mathrm{d} x)_{e}^{2}$, led to a series of experiments in the Kellogg Radiation Laboratory at Caltech by Joseph Griffith ${ }^{4}$ and Elizabeth Seiberling 5 . These workers utilized beams of heavy ions $\left({ }^{16} \mathrm{O},{ }^{19} \mathrm{~F},{ }^{20} \mathrm{Ne}\right)$ to bombard a dielectric target, $\mathrm{UF}_{4}$. Sputtered ${ }^{235} \mathrm{U}$ atoms were collected on mica, fissioned with thermal neutrons, and the resulting fission damage tracks in the mica counted to determine the yield $S$. This sensitive technique $^{6}$ has allowed the Caltech group to determine the energy spectrum of sputtered particles as well as the dependence on bombarding energy. They discovered that, for ${ }^{19} \mathrm{~F}$ on $\mathrm{UF}_{4}$, the excitation curve had a much steeper dependence on energy than $(\mathrm{d} E / \mathrm{d} x)_{e}^{2}$, and that the energy spectrum of sputtered particles was essentially thermal at a temperature of $3,500 \mathrm{~K}$. To explain their data Seiberling and co-workers have proposed a "thermalized' ion explosion model, wherein Coulomb energy of neighbouring ion pairs is transformed into random thermal kinetic energy of atomic motion of particles located along the ion path. The model correctly gives the strong energy dependence of the yield, as well as the energy spectrum.

P.K. Haff and L.E. Seiberling are in the W.K. Kellogg Radiation Laboratory, California Institute of Technology, Pasadena. 